\title{
Principles of a Sustainable Traffic Management
}

\author{
Manfred Boltze \\ Technische Universität Darmstadt \\ Otto-Berndt-Str. 2, 64287 Darmstadt, Germany \\ boltze@verkehr.tu-darmstadt.de
}

All over the world, transport demand and transport systems are changing rapidly, bringing up major challenges to deal with already existing problems and to maintain sustainability. Congestion, climate change, noise and air pollution, health impacts, traffic safety, accessibility and social inclusion, affordability and financial constraints are major problems which need to be addressed. On the other hand, new approaches in transport planning and traffic engineering as well as new technologies - e.g. information and communication systems and driverless vehicles - are enabling new transport services which can contribute significantly to solve current problems.

To set up a sustainable traffic management, it is necessary to follow some principles which must be reflected in all levels, from transport policy to traffic engineering. This presentation will outline such principles based on international experience and on a clear definition of goals and objectives in traffic management. The principles will address the need to control demand and modal choice, and the role of mobility pricing will be highlighted. Changing conditions and requirements will require a flexible use of the built infrastructure, and operation of our transport systems must become more dynamic and situation-responsive. New concepts of mobility and the application of new technologies must be promoted. Beyond traffic safety and environmental issues, the impacts of traffic on human health must gain more attention. As major preconditions of a sustainable traffic management, the right institutional framework must be implemented, and sufficient financing must be secured. Finally, the quality of traffic must become transparent and needs to be improved continuously. 